EPJ Web of Conferences 21, 05001 (2012)

DOI: 10.1051/epjconf/20122105001

(C) Owned by the authors, published by EDP Sciences, 2012

\title{
Recent experimental results on level densities for compound reaction calculations
}

\author{
A.V. Voinova , S.M. Grimes, C.R. Brune, T. Massey, and A. Schiller \\ Physics and Astronomy Department, Ohio University, Athens 45701, Ohio
}

\begin{abstract}
Proton evaporation spectra from ${ }^{6,7} \mathrm{Li}$ induced reactions on ${ }^{54,56,58} \mathrm{Fe}$ nuclei have been measured and analyzed with Hauser-Feshbach compound reaction calculations with a Gilbert and Cameron input level density model. Level densities of residual ${ }^{59-64} \mathrm{Ni}$ nuclei have been extracted from proton spectra. Both level density and level density parameter $a$ have been compared against the respective values obtained from neutron resonance spacing data. The discrepancy has been found in systematics behavior of level densities against the mass number of $\mathrm{Ni}$ isotopes.
\end{abstract}

\section{Introduction}

There is a general problem related to level density inputs for Hauser-Feshbach model calculations. Modern computer codes have several options to choose from but it is not clear which of them has to be used in some particular cases. Availability of many options helps to describe existing experimental data but it creates problems when it comes to predictions. Traditionally, different level density systematics are based on experimental data from neutron resonance spacings which are available for limited spin interval and one parity only. On the other hand reaction cross section calculations use the level density for all spins (total level density) and both parities. This can potentially create large uncertainties when converting the neutron resonance spacing to the total level density since the spin cutoff factor is not known experimentally at the neutron separation energy. It is obvious that experimental information on total level density obtained from neutron resonance spacing data needs validation from other type of experiments. It is known that the total level density can be obtained from energy spectra of outgoing particles from compound nuclear reactions. However, experimental data are scarce and no any systematic conclusions have been drawn so far.

We measured proton evaporation spectra from ${ }^{6,7} \mathrm{Li}$ induced reactions on ${ }^{54,56,58} \mathrm{Fe}$ isotopes. First generation protons populate ${ }^{59-64} \mathrm{Ni}$ nuclei for which the level density can be studied from proton spectra. Because neutron resonance data are available for almost all Ni isotopes $\left({ }^{59,60,61,62,63,65} \mathrm{Ni}\right)$ studied in this work from lithium induced reactions, the main idea of this work is to compare the total level densities obtained from nuclear reactions and those calculated from neutron resonance data. Experimental data are presented.

\section{Experiment and results}

We measured proton spectra from $15 \mathrm{MeV}$ lithium induced reactions on ${ }^{54,56,58} \mathrm{Fe}$ targets. The beam was produced by the Edwards Laboratory tandem electrostatic accelerator. The targets foils were enriched iron isotopes of about $0.5 \mathrm{mg} / \mathrm{cm}^{2}$ thickness placed in the center of the target chamber. The proton spectra were measured at backward 157 and 146 degrees angles. The low energy protons

a e-mail: voinov@ohio.edu

This is an Open Access article distributed under the terms of the Creative Commons Attribution-Noncommercial License 3.0, which permits unrestricted use, distribution, and reproduction in any noncommercial medium, provided the original work is properly cited. 

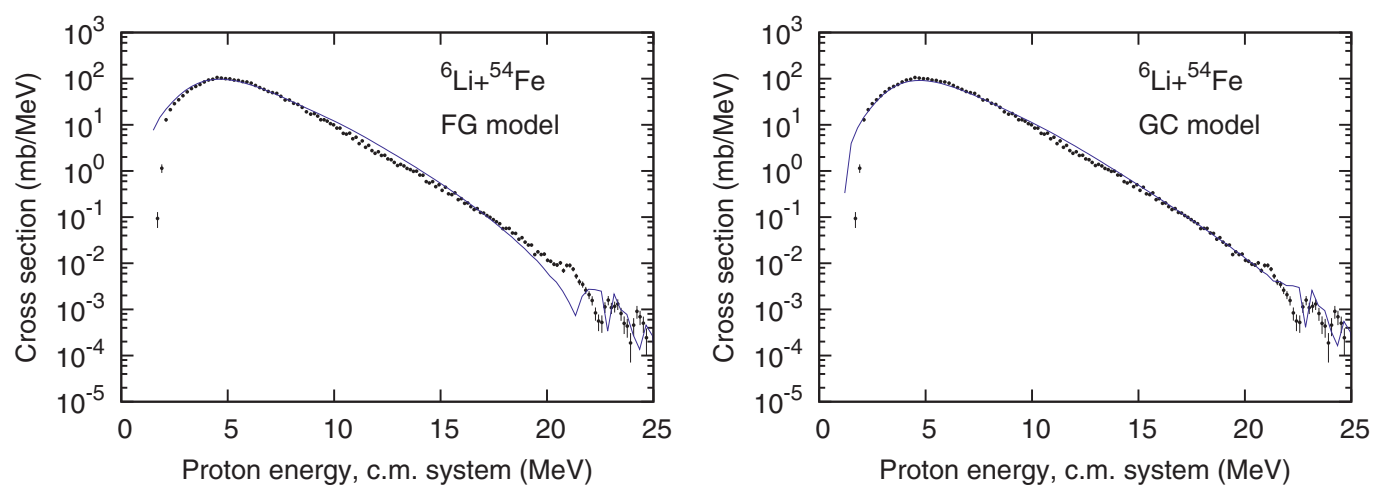

Fig. 1. Proton evaporation spectra from the ${ }^{6} \mathrm{Li}+{ }^{54} \mathrm{Fe}$ reaction. Points are experiment, lines are calculations with FG (left panel) and GC(right panel) models

( from 2 to $10 \mathrm{MeV}$ ) were selected by registering time of flight and the energy deposited in Si detectors mounted at the end of the $2 \mathrm{~m}$ and $1 \mathrm{~m}$ flight path tubes. The high energy protons (from 7 to 30 $\mathrm{MeV}$ ) were measured by $\triangle \mathrm{E}-\mathrm{E}$ Si telescope placed at the 150 degree angle in respect to the beam line. The telescope was placed at the distance of about $15 \mathrm{~cm}$ from the target to increase the solid angle. The proton spectrum was constructed from low and high energy parts matching in the region of their overlapping.

The experimental proton spectrum from the ${ }^{6} \mathrm{Li}+{ }^{54} \mathrm{Fe}$ reaction is presented in Fig. 1 along with calculations with using both Fermi-gas (FG) and Gilbert-Cameron (GC) models [4]. The GC model function consists of constant temperature and Fermi-gas parts matching at the excitation energy around $15 \mathrm{MeV}$. One can see that GC model reproduces the spectrum well in the whole energy region while the FG model has a different slope in the high energy region. We conclude that the general form of the FG model does not reproduce data in the whole energy region. This is in agreement with our early finding [1] that the constant temperature level density model gives a better agreement with an experiment in this mass range.

The proton spectra from all reactions are shown in Fig. 2 along with calculations with the GC model. The parameter systematics for GC model has been used from the Empire computer code [2] as that cited in Ref.[3]. This systematics is based upon experimental data on discrete low-lying levels and neutron resonance spacings. Calculations have been scaled up or down within 20 percents to compare only shapes of evaporation spectra determined mainly by the shape of the level density function of corresponding residual $\mathrm{Ni}$ isotopes. The absolute scale is not the focus of this work since it is determined by several other factors including fusion cross section and competition between different outgoing channels due to different level densities of corresponding residual nuclei. The low energy part of some of the spectra is not reproduced well due to the problems with calculations of the second stage protons from the $(\mathrm{Li}, \mathrm{np})$ reaction channel.

One can see from Fig. 2 that the slope of experimental proton spectra is consistent for all reactions. However, it increases in calculations as the neutron number of the residual $\mathrm{Ni}$ isotopes increases. The noticeable difference occurs for the spectra from ${ }^{6,7} \mathrm{Li}$ on ${ }^{58} \mathrm{Fe}$ reactions. It obviously results from the difference of corresponding level density functions of residual $\mathrm{Ni}$ isotopes populated by first stage protons. Since the global level density systematics was used in calculations, it is important to check the consistency of the total level densities for local Ni isotopes. For this purpose we extracted total level densities from proton evaporation spectra and plotted them against GC functions fitted to lowlying discrete levels and neutron resonance spacings (Fig.3). Parameters of the GC model have been adjusted individually for each ${ }^{59-64} \mathrm{Ni}$ isotopes and plotted versus mass number in Fig.4. The figure shows that the $a$ parameter adjusted to the total level density from proton spectra has a weaker A dependence compared to ones adjusted to neutron resonance spacings.

The different behavior of the $a$ parameter obtained from neutron resonance spacings versus one from proton evaporation spectra may indicate that the spin cutoff parameter used to convert neutron 
$\mathrm{CNR} * 11$
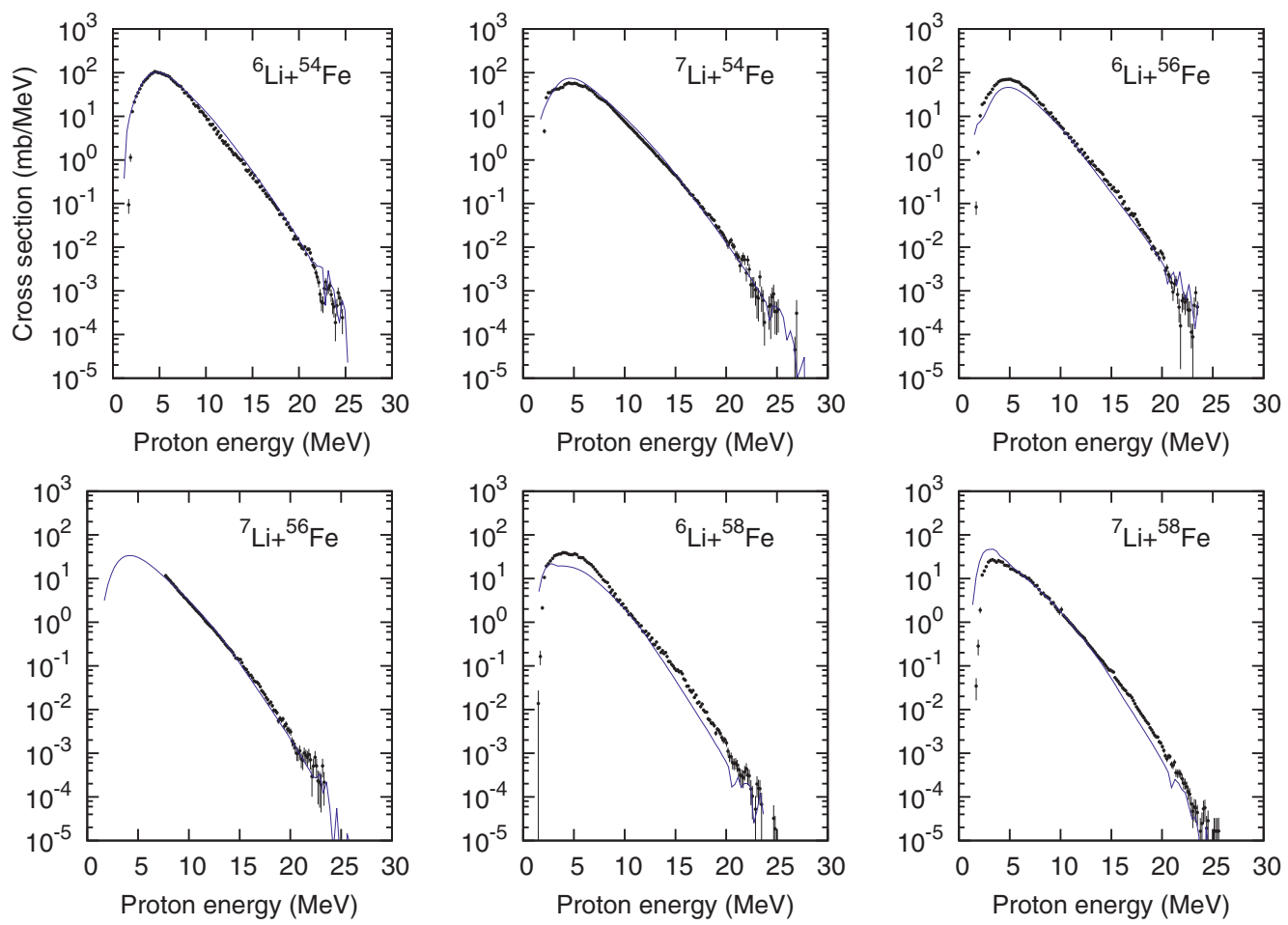

Fig. 2. Proton evaporation spectra. Points are experiment, lines are calculations with GC level density model.
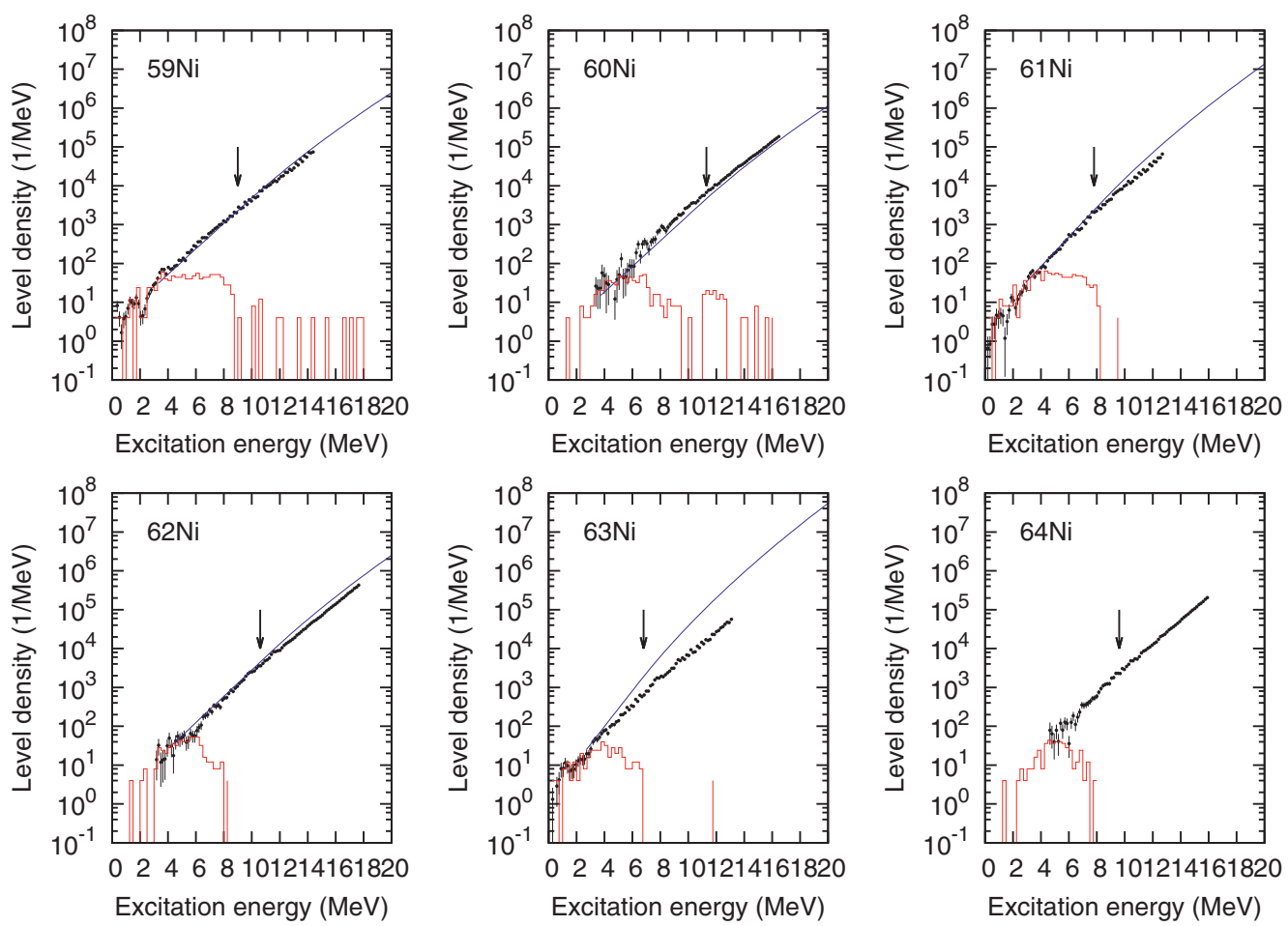

Fig. 3. Level densities obtained from proton evaporation spectra (points) and from neutron resonance spacings (lines). Histograms are densities of discrete levels. Arrows indicate the neutron binding energy. 


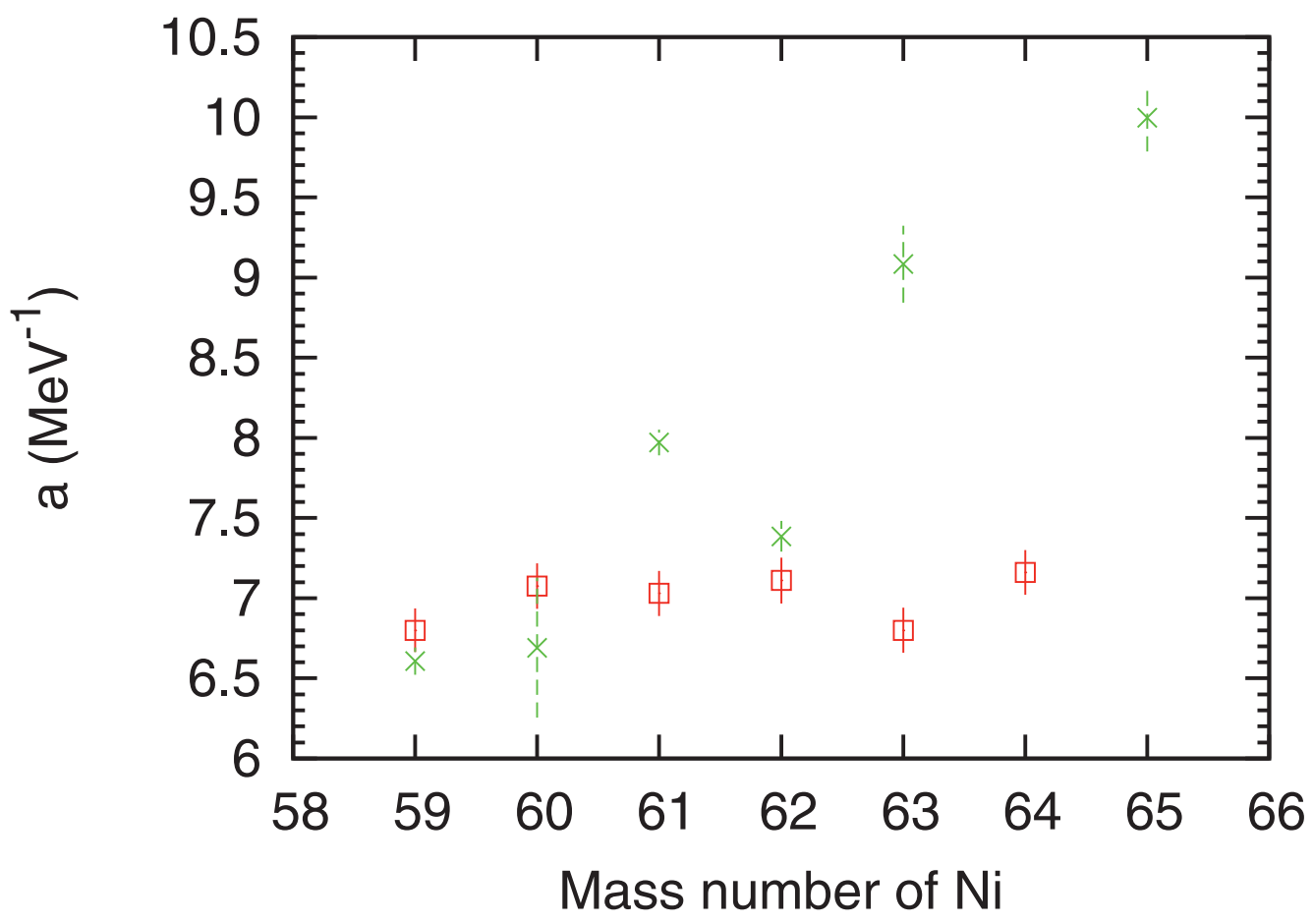

Fig. 4. Level density parameter $a$ obtained from both neutron resonance data (stars) and from proton evaporation spectra (squares)

resonance spacings to the total level density does not follow the assumed simple Fermi-gas model when the mass number of $\mathrm{Ni}$ isotopes increases. The assumption about the equality of negative and positive parities can also be incorrect. It seems that the shell effects which play important role in the A dependence of the $a$ parameter if we get it from neutron resonance spacings, has a little influence on total level densities obtained from proton evaporation spectra. It means that new level density systematics which would be based on particle evaporation spectra from compound nuclear reactions is needed. It would help to solve problems with level density inputs in Hauser-Feshbach reactions cross section calculations.

\section{References}

1. A. V. Voinov, B. M. Oginni, S. M. Grimes, C. R. Brune, M. Guttormsen, A. C. Larsen, T. N. Massey, A. Schiller and S. Siem, Phys.Rev. C79,(2009) 031301(R)

2. M. Herman, R. Capote, B. Carlson, P. Obložinský, M. Sin, A. Trkov, H. Wienke, V. Zerkin, Nucl. Data Sheets 108, (2007) 2655

3. P. G. Young, E. D. Arthur, M. Bozoian, T. R. England, G. M. Hale, R. J. LaBauve, R. C. Little, R. E. MacFarlane, D. G. Madland, R. T. Perry, W. B. Willson, Trans. Amer. Nucl. Soc. 60, (1989) 271

4. R. Capote, M. Herman, P. Oblozinsky, P.G. Young, S. Goriely, T. Belgya, A.V. Ignatyuk, A.J. Koning, S. Hilaire, V.A. Plujko, M. Avrigeanu, O. Bersillon, M.B. Chadwick, T. Fukahori, Zhigang Ge, Yinlu Han, S. Kailas, J. Kopecky, V.M. Maslov, G. Reffo, M. Sin, E.Sh. Soukhovitskii, P. Talou, Nucl. Data Sheets 110, (2009) 3107 